\title{
Problematic smartphone use in young Swiss men: Its association with problematic substance use and risk factors derived from the pathway model
}

\author{
MICHELLE DEY ${ }^{1 *}$, JOSEPH STUDER ${ }^{2}$, MICHAEL PATRICK SCHAUB ${ }^{1}$, GERHARD GMEL $^{2}$, \\ DAVID DANIEL EBERT ${ }^{3}$, JENNY YI-CHEN LEE ${ }^{1}$ and SEVERIN HAUG ${ }^{1}$ \\ ${ }^{1}$ Swiss Research Institute for Public Health and Addiction, University of Zurich, Zurich, Switzerland \\ ${ }^{2}$ Alcohol Treatment Centre, Lausanne University Hospital CHUV, Lausanne, Switzerland \\ ${ }^{3}$ Department of Clinical Psychology and Psychotherapy, Friedrich-Alexander University Erlangen-Nürnberg, Erlangen, Germany
}

(Received: December 20, 2018; revised manuscript received: March 28, 2019; accepted: April 6, 2019)

\begin{abstract}
Background and aims: This study aimed to examine associations between risk factors suggested in the pathway model proposed by Billieux et al., demographic and substance use variables, and problematic smartphone use (PSU). Methods: The analytical sample consisted of 5,096 Swiss men (mean age $=25.5$ years, $S D=1.26$ ). Multiple linear regression analyses were conducted with PSU as dependent and the following as independent variables: (a) Billieux's pathway model variables (depression, social anxiety, ADHD, aggression-hostility, and sensation seeking); (b) substance use variables [alcohol: at-risk risky single-occasion drinking (RSOD); at-risk volume drinking; tobacco use: daily smoking; illicit drug use: more than weekly cannabis use; having used at least one other illicit drug besides cannabis over the preceding 12 months]; and (c) sociodemographic variables (age, language region, and education). Results: All pathway-model variables except sensation seeking were significant predictors of PSU, especially symptoms of social anxiety $(\beta=0.196)$ and ADHD $(\beta=0.184)$. At-risk RSOD was positively $(\beta=0.071)$ associated with PSU, whereas both frequent cannabis use $(\beta=-0.060)$ and daily cigarette smoking $(\beta=-0.035)$ were negatively associated with PSU. Higher-achieved educational levels and being from the Germanspeaking part of Switzerland predicted PSU. Discussion and conclusions: The findings of this study can be used to develop tailored interventional programs that address the co-occurrence of certain risky behaviors (e.g., at-risk RSOD and PSU) and target individuals who might be particularly prone to PSU. Such interventions would need to ensure that addressing one problem (e.g., decreasing PSU) does not lead to some other compensatory behavior (e.g., frequent cigarette smoking).
\end{abstract}

Keywords: problematic smartphone use, smartphone addiction, pathway model, substance use, representative sample, Switzerland

\section{INTRODUCTION}

Smartphones perform many of the functions of a computer (Oxford Dictionary, 2018) but are even more convenient, allowing users to engage in a range of activities, like surfing the Internet, e-mailing, and social networking almost anywhere. However, potential negative consequences of their use are being increasingly discussed (Billieux, 2012). Some authors have even conceptualized the maladaptive use of smartphones as a behavioral addiction, due to similarities for example, impaired control over one's use (Lin et al., 2016) - with substance-related and recognized nonsubstance-related addictive disorders (e.g., gambling disorder). This being said, smartphone addiction has not yet been included as a formal diagnosis in the ICD-11 draft or DSM-5 (Panova \& Carbonell, 2018). Accordingly, it has been argued that the consequences of excessive smartphone use might not meet the severity levels of an addiction (Panova \& Carbonell, 2018). Furthermore, evidence that either confirms or rejects the conceptualization of maladaptive smartphone use as a behavioral addiction remains scarce, particularly research revealing similarities between mobile phone addiction and other widely recognized addictive behaviors (Billieux, Maurage, Lopez-Fernandez, Kuss, \& Griffiths, 2015). Therefore, it has been proposed that the term "smartphone addiction" should be replaced with "problematic smartphone use" (PSU), at least for time being (Panova \& Carbonell, 2018). This former term will be used for the duration of the current paper.

PSU has been defined as "an inability to regulate one's use of the smartphone, which eventually involves negative consequences in daily life" (Billieux, 2012). According to a model proposed by Billieux et al. (2015), PSU can be driven (a) by an individual's strong need to maintain relationships and obtain reassurance from others (excessive reassurance

\footnotetext{
* Corresponding author: Michelle Dey; Swiss Research Institute for Public Health and Addiction, University of Zurich, Konradstrasse 32, 8005 Zurich, Switzerland; Phone: +41 4444811 90; Fax: +41 4444811 70; E-mail: michelle.dey@isgf.uzh.ch
}

This is an open-access article distributed under the terms of the Creative Commons Attribution-NonCommercial 4.0 International License, which permits unrestricted use, distribution, and reproduction in any medium for non-commercial purposes, provided the original author and source are credited, a link to the CC License is provided, and changes - if any - are indicated. 
pathway); (b) by a person's poor impulse control, resulting in uncontrolled urges and deregulated use (impulsiveantisocial pathway); or (c) by a strong and constant desire to communicate with others and establish new relationships, as well as a steady need for stimulation and a high sensitivity to rewards (extraversion pathway). While all three pathways might cause an addictive pattern of smartphone use, the impulsive-antisocial and extraversion pathways can also lead to antisocial or risky behavioral patterns (Billieux et al., 2015). These include prohibited and inappropriate use of smartphones such as driving while using cell phones. Furthermore, it must be acknowledged that these pathways, which are not mutually exclusive, all have their unique risk factors. Low self-esteem, emotional instability, or neuroticism have, for instance, been proposed as risk factors for the excessive reassurance pathway; emotion laden impulsivity, aggressive traits, or attention-deficit hyperactivity disorder (ADHD) symptoms for the impulsiveantisocial pathway; and extraversion, sensation seeking, or reward sensitivity for the extraversion pathway.

Various studies provide empirical evidence for the link between risk factors proposed in the pathway model and PSU. Regarding the excessive reassurance pathway, the associations between depression or anxiety and PSU are particularly well-established (Elhai, Dvorak, Levine, \& Hall, 2017; Vahedi \& Saiphoo, 2018). In this case, the PSU might not only be interpreted as being induced by a strong need to maintain relationships and obtain reassurance from others, but also as a way for anxious or depressed people to cope and distract themselves from negative emotions (Elhai et al., 2017). In terms of the impulsiveantisocial pathway, impulsivity has been studied frequently. Among other things, it has been shown that dysfunctional impulsivity - defined as a tendency to act with less forethought - was a predictor for a smartphone addiction predisposition (Kim et al., 2016). Furthermore, studies revealed significant associations between particular facets of impulsivity (e.g., urgency) and perceived dependence on the cellular phone (Billieux, Van der Linden, D'Acremont, Ceschi, \& Zermatten, 2007) or problematic use (Billieux, Van der Linden, \& Rochat, 2008). Finally, a study that combined measures of behavior and electrophysiology (event-related potentials) found deficits in the early stage of inhibition processing among excessive smartphone users (Chen, Liang, Mai, Zhong, \& Qu, 2016). Some empirical evidence for the risk factors suggested in the extraversion pathway exists as well. For instance, it has been found that extraversion is a significant predictor for nomophobia (i.e., the fear or worry at the idea of being without one's mobile phone or unable to use it; Cambridge Dictionary, 2019; Argumosa-Villar, Boada-Grau, \& Vigil-Colet, 2017) and sensation seeking for a smartphone application-based addiction (Csibi, Griffiths, Cook, Demetrovics, \& Szabo, 2018).

Despite the extensive empirical evidence for some risk factors (e.g., depression, anxiety, and impulsivity) suggested in the pathway model, it needs to be acknowledged that other risk factors such as ADHD symptoms have not yet gained as much attention. In addition, most published empirical research used convenient samples. The study currently being presented aimed to fill these gaps by studying a variety of risk factors in an explorative manner for the different pathways leading to PSU, and by relying on a representative sample of young Swiss men.

The second aim of this study was to identify associations between substance use and PSU. More precisely, we evaluated associations between risky alcohol consumption and frequent cigarette smoking and PSU, since similarities between substance use and PSU might only appear when such extreme substance use patterns are considered. Furthermore, we sought associations between frequent cannabis use, as well as the use of other illicit drugs and PSU, since such associations have not yet been studied; only associations with problematic/ intensive cellular phone use have been examined previously (Sánchez-Martínez \& Otero, 2009; Yang, Yen, Ko, Cheng, \& Yen, 2010).

\section{METHODS}

\section{Procedures}

For this study, data from the "Cohort Study on Substance Use Risk Factors" (C-SURF) - a longitudinal study with a particular focus on substance-related risk and protective factors - were used. Participants were enrolled between August 23, 2010 and November 11, 2011 at three of the six army-recruitment centers in the German- and Frenchspeaking parts of Switzerland, covering 21 of the country's 26 existing cantons. In Switzerland, all men undergo a mandatory army-recruitment process at roughly 19-20 years old to assess their eligibility for military or civil service; as such, there is no preselection. Hence, virtually all men in this age group and from the 21 participating cantons were eligible for study inclusion. During enrollment, 7,556 young men gave written consent to participate in the study. Even though study enrollment took place at army-recruitment centers, C-SURF is independent of the army. Young men who consented to participate in the study were invited by mail or e-mail to complete either a paper-and-pen or online questionnaire, per their preference. Cross-sectional data from the third wave of data collection were considered for this study. No longitudinal data were analyzed because the dependent variable used in the current analyses as well as some independent variables were only assessed in the third wave of data collection.

\section{Participants and the analytical sample}

Altogether, 5,516 participants filled out the third-wave questionnaire between April 28, 2016 and March 26, 2018 (response rate $=73 \%$; for more details, see Gmel et al., 2015 or www.c-surf.ch). Among these 5,516 participants, 242 did not own a smartphone and 6 men did not answer the question about smartphone ownership. These 248 men were excluded from further analysis. Furthermore, 172 people were excluded due to missing data for one of the variables of interest (e.g., regarding PSU). Hence, the final analyzed sample consisted of 5,096 men. 


\section{Measures}

PSU (dependent variable): Smartphone Addiction Scale Short Version (SAS-SV). The SAS-SV (Kwon, Kim, Cho, \& Yang, 2013) was used to assess PSU. The SAS-SV is a selfreport measurement tool that consists of 10 items (derived from the original SAS; Kwon, Lee, et al., 2013). All 10 items are rated on a 6-point Likert scale, ranging from 1 "strongly disagree" to 6 "strongly agree." The total SASSV score ranges from 10 to 60 , with higher scores indicating more PSU. The German version we used was based on the translation of Haug et al. (2015), although Items 7 and 8 were adapted to correspond more closely to the English translation in Kwon, Kim, et al. (2013). The French version was developed by the French-speaking members of the CSURF team. Cronbach's $\alpha$ was .88 in this study, and .91 in the original study from Korea (Kwon, Kim, et al., 2013). Some of these previously published investigators (Alhazmi, Alzahrani, Baig, Salawati, \& Alkatheri, 2018; Chen et al., 2017; Haug et al., 2015; Kwon, Kim, et al., 2013) employed a particular cut-off to dichotomize the total SAS-SV into "non-PSU" vs. "PSU." However, the threshold they used for men $(\geq 31)$ might not be adequate for the current sample, since these previous studies targeted younger samples (Haug et al., 2015; Kwon, Kim, et al., 2013) or were orchestrated in Asia (Chen et al., 2017; Kwon, Kim, et al., 2013) or Saudi Arabia (Alhazmi et al., 2018). Furthermore, PSU was not conceptualized as a disorder in this study, but rather as a behavior that ranges from unproblematic to problematic. Hence, we used a continuous variable for all related analyses.

Independent variables

Predictors from the Billieux model. As mentioned in "Introduction" section, the model that Billieux et al. (2015) proposed was used to guide the selection of predictors, in which subsequently each was allocated to one of the three suggested pathways. Several indicators that could be interpreted as risk factors for the excessive reassurance pathway have been assessed in the third wave of data collection. However, to avoid multicollinearity, only two predictors were included in the present analyses. First, self-reported depressive symptoms were assessed with the Major Depression Inventory (WHO-MDI; Bech, Rasmussen, Olsen, Noerholm, \& Abildgaard, 2001; Olsen, Jensen, Noerholm, Martiny, \& Bech, 2003). On a 6-point Likert scale, each of the 10 items measures how often a particular symptom has been experienced over the preceding 14 days, ranging from 0 "at no time" to 5 "all the time." Two of these items are divided into two subitems, $a$ and $b$, whereby only the ones with the higher score (either a or b) were considered (Olsen et al., 2003). Hence, the summation score utilized in our analyses has a theoretical range of 0-50. Second, the selfreported Clinically Useful Social Anxiety Disorder Outcome Scale (CUSADOS; Dalrymple et al., 2013) was included. Its 12 items assess symptoms of social anxiety over the past week, all rated on a 5-point Likert scale, ranging from 0 "not at all true" to 4 "almost always true." A summation score of these items was used (theoretical range: 0-48).

The impulsive-antisocial pathway was covered by two risk factors. First, ADHD was assessed using the World
Health Organization (WHO) Adult ADHD Self-Report Scale Screener (ASRS-v1.1; Kessler et al., 2005, 2007). This instrument's six questions are based on DSM-IV diagnostic criteria for ADHD and - in C-SURF's third wave - refer to the preceding 12 months. Each item is rated on a 5-point Likert scale, ranging from 0 (never) to 4 (very often). The summation score has a theoretical range of 0-24. Second, the antisocial component of the pathway was assessed using the aggression-hostility (Agg-Host) subscale of the short form of the Zuckerman-Kuhlman Personality Scale (ZKPQ-50-cc; Aluja et al., 2006). This subscale consists of 10 self-report statements, all of which must be rated as being either true $(1$, indicating the trait) or false (0). The instrument's three reversely formulated items ("false" indicating the trait) were recoded. We used the summation score of the 10 items, with a theoretical range between 0 and 10 .

For the extraversion pathway, C-SURF assessed sensation seeking with the Brief Sensation Seeking Scale (BSSS; Hoyle, Stephenson, Palmgreen, Lorch, \& Donohew, 2002). This selfreport measurement consists of eight items, each rated on a 5-point Likert scale, ranging from 1 (strongly disagree) to 5 (strongly agree). The mean of these eight items was used for the current paper (theoretical range $=1-5$ ), with higher scores indicating higher levels of sensation seeking.

(At-risk) substance use (over the past 12 months). Besides the predictors suggested as risk factors by Billieux et al. (2015), the following risky substance use variables were considered:

- for alcohol:

- At-risk risky single-occasion drinking (RSOD): RSOD was defined as consuming at least six standard drinks on a single occasion (pictures of standard drinks containing 10-12 g of pure alcohol were shown for reference). At-risk RSOD was defined as RSOD at least monthly (coded as 1). In contrast, not at-risk RSOD (coded as 0) included men who (a) reported RSOD, but less than once monthly; (b) drank alcohol, but exhibited no RSOD; or (c) were abstinent.

- At-risk volume drinking: At-risk volume drinkers included those who reported drinking at least 21 drinks per week (coded as 1); whereas those who reported drinking less (including abstinent participants) were classified as not at-risk volume drinkers (coded as 0).

- Daily cigarette smoking: Daily cigarette smokers (coded as 1) were differentiated from those who did not smoke daily (including occasional as well as nonsmokers; coded as 0).

- More than weekly cannabis use: More than weekly cannabis users (coded as 1) were differentiated from those who reported using cannabis less frequently (including those who claimed not to use cannabis at all; coded as 0 ).

- Using at least one illicit drug other than cannabis: The use of illicit drugs (natural hallucinogens, other synthetic hallucinogens, salvia divinorum, amphetamine/ speed, amphetamine sulfate, khat, methamphetamine, poppers, solvent sniffing, ecstasy, cocaine, crack, freebase, heroine, morphine, opium, ketamine, DXM, methadone, GHB/GBL/1-4 butanediol, "bath salts," 
"research chemicals," or legal highs, spice or similar substances containing synthetic cannabinoids, ayahuasca/DMT, psychoactive plants from the rainforest, and ibogaine) was dichotomized into "no use" (coded as 0) versus "using at least one illicit drug other than cannabis" (coded as 1) over the preceding 12 months.

Sociodemographic variables. Participant age, language region (French- vs. German-speaking), and highest achieved level of education were included as demographic variables, the last with the following response options: "mandatory schooling," "vocational schooling," "high school/ professional baccalaureate," and "university/university of applied sciences (bachelor/master)."

\section{Statistical analyses}

All analyses were conducted using SPSS version 22.0 (IBM Corp., 2013). Linear regression analyses (simple and multiple) were conducted with PSU (as a continuous variable) as the dependent variable and the following as independent: all variables extracted from the model proposed by Billieux et al. (2015), the above-listed substance use variables, and the three above-listed sociodemographic variables. Logarithmic transformation was applied to the SAS-SV score (outcome) to improve the normal distribution of residuals. For multiple regression analyses, with all independent variables considered in the model concurrently, variance inflation factors (VIF) were used to assess whether multicollinearity between the independent variables existed. A VIF $\geq 10$ was interpreted as indicating the presence of multicollinearity (Myers, 1990).

\section{Ethics}

The study protocol was approved by the Ethics Committee for Clinical Research at Lausanne University Medical School (protocol number: 15/07).

\section{RESULTS}

The sample characteristics and descriptive statistics for all included dependent and independent variables are summarized in Table 1. The results of simple and multiple regression analyses are summarized in Table 2. No indicators of multicollinearity were present (all VIF factors $\leq 10$ ). All independent variables for the excessive reassurance pathway (WHO-MDI and CUSADOS), impulsive-antisocial pathway (ASRS-v1.1 and Agg-Host), and extraversion pathway (BSSS) were significant predictors of PSU in simple regression models. Similar results were obtained for a multiple regression model that included all the independent variables (including substance use and sociodemographic variables), although only a trend $(.05<p<.10)$ was identified for BSSS. Simultaneously, considering all predictors, CUSADOS and ASRS-v1.1 were the most important predictors for PSU, as indicated by the highest standardized $\beta$.

Concerning substance use, at-risk RSOD, at-risk volume drinking, and the use of at least one illicit drug besides cannabis were significant predictors of PSU on simple
Table 1. Sample characteristics and descriptive statistics for all variables considered

\begin{tabular}{lc}
\hline & $\begin{array}{c}\text { Total analytical } \\
\text { sample }(n=5,096)\end{array}$ \\
\hline Independent variable for regression & \\
analyses & \\
SAS-SV (log-transformed sum & $2.83(0.40)$, \\
score): mean $(S D)$, range & $2.30-4.01$ \\
Dependent variables for regression & \\
analyses & \\
WHO-MDI: mean $(S D)$, range & $8.58(7.29), 0-47.0$ \\
CUSADOS: mean $(S D)$, range & $7.47(8.18), 0-48.0$ \\
ASRS-v1.1: mean $(S D)$, range & $6.88(4.18), 0-24.0$ \\
Agg-Host: mean $(S D)$, range & $3.78(2.15), 0-10.0$ \\
BSSS: mean $(S D)$, range & $3.00(0.80), 1.0-5.0$ \\
At-risk RSOD: $n(\%)$ & $1,958(38.4)$ \\
At-risk volume drinking: $n(\%)$ & $351(6.9)$ \\
Daily cigarette smoking: $n(\%)$ & $1,070(21.0)$ \\
More than weekly cannabis use: $n(\%)$ & $411(8.1)$ \\
Using at least one illicit drug other & $692(13.6)$ \\
than cannabis: $n(\%)$ & \\
Age: mean $(S D)$ & $25.5(1.26)$ \\
French-speaking part of Switzerland: & $2,902(56.9)$ \\
$n(\%)$ & \\
Highest achieved education & \\
Mandatory schooling: $n(\%)$ & $161(3.2)$ \\
Vocation schooling: $n(\%)$ & $2,046(40.1)$ \\
High school/professional & $1,242(24.4)$ \\
baccalaureate: $n(\%)$ & $1,647(32.3)$ \\
University/university of applied & \\
$\quad$ sciences (bachelor/master): $n(\%)$ & \\
\hline
\end{tabular}

Note. SD: standard deviation; Agg-Host: aggression-hostility subscale from the Zuckerman-Kuhlman Personality Scale; ASRSv1.1: Adult ADHD Self-Reported Scale Screener; BSSS: Brief Sensation Seeking Scale; CUSADOS: Clinically Useful Social Anxiety Disorder Outcome Scale; RSOD: risky single-occasion drinking; SAS-SV: Smartphone Addiction Scale - Short Version; WHO-MDI: Major Depression Inventory.

regression analysis. However, only at-risk RSOD remained significant in the multiple regression model that included all the independent variables. More than weekly cannabis use and daily cigarette smoking were negatively associated with PSU when the model was adjusted for all other independent variables.

Finally, some sociodemographic variables were identified as significant predictors of PSU. On multiple regression analysis, those reporting either one of the two highest education levels - "high school/ professional baccalaureate" or "university/university of applied sciences (bachelors/ master's degree)" - had a higher mean PSU score than those with "mandatory schooling." Moreover, those from the French-speaking part of Switzerland had lower PSU scores than those from the German-speaking part.

\section{DISCUSSION}

In the study reported here, we evaluated PSU predictors in a representative sample of young Swiss men. All selected 
Table 2. Simple and multiple linear regression analyses on problematic smartphone use

\begin{tabular}{|c|c|c|c|c|c|c|}
\hline & \multicolumn{3}{|c|}{ Simple linear regression } & \multicolumn{3}{|c|}{ Multiple linear regression ${ }^{\mathrm{a}}$} \\
\hline & $B$ & $S E B$ & $\beta$ & $B$ & $S E B$ & $\beta$ \\
\hline \multicolumn{7}{|l|}{ Excessive reassurance pathway } \\
\hline WHO-MDI & 0.012 & 0.001 & $0.221 * * *$ & 0.003 & 0.001 & $0.061 * * *$ \\
\hline CUSADOS & 0.014 & 0.001 & $0.288 * * *$ & 0.010 & 0.001 & $0.196 * * *$ \\
\hline \multicolumn{7}{|l|}{ Impulsive-antisocial pathway } \\
\hline ASRS-v1.1 & 0.028 & 0.001 & $0.291 * * *$ & 0.018 & 0.001 & $0.184 * * *$ \\
\hline Agg-Host & 0.026 & 0.003 & $0.138 * * *$ & 0.017 & 0.002 & $0.092 * * *$ \\
\hline \multicolumn{7}{|l|}{ Extraversion pathway } \\
\hline BSSS & 0.049 & 0.007 & $0.099 * * *$ & 0.014 & 0.007 & $0.028^{\#}$ \\
\hline \multicolumn{7}{|l|}{ (At-risk) substance use } \\
\hline At-risk RSOD & 0.087 & 0.011 & $0.107 * * *$ & 0.059 & 0.012 & $0.071 * * *$ \\
\hline At-risk volume drinking & 0.071 & 0.022 & $0.045^{* * *}$ & 0.007 & 0.022 & 0.005 \\
\hline Daily cigarette smoking & -0.021 & 0.014 & -0.021 & -0.034 & 0.014 & $-0.035^{*}$ \\
\hline More than weekly cannabis use & -0.026 & 0.021 & -0.018 & -0.088 & 0.021 & $-0.060 * * *$ \\
\hline Using at least one illicit drug other than cannabis & 0.074 & 0.016 & $0.064 * * *$ & 0.022 & 0.017 & 0.019 \\
\hline \multicolumn{7}{|l|}{ Sociodemographic variables } \\
\hline Age & -0.002 & 0.004 & -0.007 & 0.002 & 0.004 & 0.006 \\
\hline French-speaking part of Switzerland & 0.019 & 0.011 & $0.024^{\#}$ & -0.022 & 0.011 & $-0.028^{*}$ \\
\hline \multicolumn{7}{|l|}{ Highest achieved education } \\
\hline Mandatory schooling (reference) & & & & & & \\
\hline Vocation schooling & 0.006 & 0.033 & 0.008 & 0.025 & 0.030 & 0.031 \\
\hline High school/professional baccalaureate & 0.063 & 0.033 & $0.068^{\#}$ & 0.064 & 0.031 & $0.069 *$ \\
\hline University/university of applied sciences (bachelor/master) & 0.077 & 0.033 & $0.091^{*}$ & 0.090 & 0.031 & $0.105 * *$ \\
\hline
\end{tabular}

Note. SE: standard error; Agg-Host: aggression-hostility subscale from the Zuckerman-Kuhlman Personality Scale; ASRS-v1.1: Adult ADHD Self-Reported Scale Screener; BSSS: Brief Sensation Seeking Scale; CUSADOS: Clinically Useful Social Anxiety Disorder Outcome Scale; RSOD: risky-single occasion drinking; WHO-MDI: Major Depression Inventory.

${ }^{\mathrm{a}}$ In the multiple regression analysis, all independent variables were included. A significant regression equation was found $[F(15$, $5080)=62.233, p<.001]$, with an $R^{2}$ of .155 .

${ }^{\#} p \leq .10$ (trend). ${ }^{*} p \leq .05 .{ }^{* *} p \leq .01 .{ }^{* * *} p \leq .001$.

variables that had been extracted from the pathway model proposed by Billieux et al. (2015) - especially symptoms of social anxiety and ADHD - were significant predictors on multiple regression analysis (except for sensation seeking). Regarding substance use, at-risk RSOD was positively associated with PSU, whereas daily cigarette smoking and more than weekly cannabis use were negatively associated with PSU. Finally, language region and highest achieved education were significant predictors of PSU.

Among the excessive reassurance pathway variables, both depression and social anxiety were significant PSU predictors in this study. Consistent with this, in a recent systematic review, depression severity was consistently linked to PSU (with at least medium effect sizes), as was anxiety (small effect sizes; Elhai et al., 2017). Another metaanalysis identified a small-to-medium association between smartphone use and stress and anxiety (summary effect size: $\kappa=39, r=.22, p<.001$, CI [0.17-0.28]), with a larger effect size identified in studies that examined problematic phone use ( $\kappa=17, r=.35, p<.001$, CI [0.27-0.42]; Vahedi $\&$ Saiphoo, 2018). Several explanations for these associations are suggested in the literature (for an overview, see Elhai et al., 2017). First, depressive or anxious individuals might use their smartphone to cope with their negative emotions and distract themselves. Similarly, the excessive reassurance pathway assumes that addiction-like smartphone use is the consequence of a need for reassurance, which might increase with increasing levels of depression or anxiety (Billieux et al., 2015). Second, PSU might itself cause symptoms of depression or anxiety. Accordingly, in one longitudinal study among young adults, a high frequency of mobile phone use at baseline was revealed to be a risk factor for mental health problems (including symptoms of depression) at 1-year follow-up (Thomée, Härenstam, \& Hagberg, 2011). Third, evidence supporting a vicious cycle between PSU and mental health problems exists (Jun, 2016). Accordingly, the model suggested by Elhai, Levine, and Hall (2019) proposes that symptoms of anxiety cause anxiety-related transdiagnostic factors (social isolation, fear of missing out, boredom proneness, and rumination), which in turn increase the frequency of smartphone use, that subsequently leads to PSU. PSU - in turn - increases symptoms of anxiety. As such, a negative reinforcement is proposed.

For the impulsive-antisocial pathway of the model of Billieux et al. (2015), PSU is assumed to be driven by poor impulse control, resulting in uncontrolled urges and deregulated smartphone use. In line with this, symptoms of ADHD (i.e., inattention and hyperactivity impulsivity; as measured by the ASRS-v1.1) predicted PSU in this study. To the best of our knowledge, previous studies only looked at particular components of ADHD and identified associations between inattention (Zheng et al., 2014), dysfunctional impulsivity (Kim et al., 2016), particular components of impulsivity (e.g., urgency; Billieux et al., 2007, 2008), or deficits in the early stage of inhibition processing (Chen et al., 2016) and 
(perceived) excessive mobile phone use or PSU. In addition to the symptoms of ADHD, in this study, we identified Agg-Host as a significant PSU predictor. This variable was included in the analyses, since Billieux et al. (2015) suggested that PSU might also express itself in antisocial patterns of use. In agreement with the pathway model and current results, other studies have detected associations between a type A personality (aggressive, competitive, angry, cynical, and mistrustful; Boumosleh \& Jaalouk, 2017) or aggression toward others (Yang et al., 2010) and PSU or problematic cellular use, respectively.

Sensation seeking was selected for the extraversion pathway (Billieux et al., 2015) in the current analyses. This variable was a significant predictor on simple regression analysis, confirming earlier findings (Csibi et al., 2018). However, only a trend was identified upon multiple regression analysis. Accordingly, another study detected no association between sensation seeking and PSU (Billieux et al., 2007). These divergent findings might possibly be due to different methodological approaches (e.g., the predictors or outcome measures included). In this regard, it must also be considered that the SAS-SV mainly adapted its items from substance-abuse questionnaires. Hence, the SAS-SV sum score used in the present article might mainly reflect an addictive form of smartphone use. Impulsivityrelated traits (including sensation seeking) have, however, typically been related to a dangerous use of the mobile phone, but less consistently to addictive use patterns (Billieux et al., 2008).

Regarding substance use, we uncovered a significant positive association between at-risk RSOD and PSU. Similarly, a number of previously published investigators have reported significant relationships between risky alcohol consumption - operationalized as increasing alcohol use scores (Choi et al., 2015), weekly to daily alcohol drinking (Luk et al., 2018), or "at least once had too much to drink/ been drunk" (Sánchez-Martínez \& Otero, 2009) - and PSU or cell phone use, respectively. In contrast, others who looked at less-risky alcohol use (dichotomized into not drinking vs. drinking alcohol) failed to discover any associations with PSU (Aker, Şahin, Sezgin, \& Oğuz, 2017; Boumosleh \& Jaalouk, 2017). These findings support the assumption that associations with PSU might only be apparent when a risky substance use behavior (excessive frequency and/or quantity) is evaluated as a potential predictor. However, not all prior studies have confirmed this conjecture (Chung et al., 2018; Haug et al., 2015; Yang et al., 2010), possibly since the definition of "at-risk consumption" might vary as a function of the age group studied and country where the study was conducted. That atrisk volume drinking was no longer a significant predictor in our multiple regression model might be, because this behavior mostly seemed to be explained by at-risk RSOD.

Previous studies that only differentiated between smokers (which might include occasional smokers) and non-smokers or between never, former, and current smokers rarely identified any association between smoking and PSU (Aker et al., 2017; Alhazmi et al., 2018; Boumosleh \& Jaalouk, 2017; Chung et al., 2018; Haug et al., 2015). However, in two representative samples from Asia, current smoking was associated with greater PSU (Luk et al., 2018), and having smoked cigarettes at least every month over the past year with problematic cellular phone use (Yang et al., 2010). Even though this study used daily smoking as a predictor (i.e., a riskier behavior that excluded occasional smokers), no significant positive association with PSU was identified. Rather, daily smoking was negatively associated with PSU on multiple regression analysis. In addition, more than weekly cannabis use was negatively associated with PSU, again on multiple regression analysis. In contrast, lifetime prevalence of marijuana/hashish use was significantly associated with intensive cellular phone use in bivariate analyses in a study conducted in Spain (Sánchez-Martínez \& Otero, 2009). In this study, the use of any other illicit drugs besides cannabis over the preceding 12 months was only a significant predictor for PSU in simple regression models. In another study involving a representative sample of Taiwanese adolescents, illicit drug use during the past year was significantly associated with problematic cell phone use in some, but not all, sociodemographic subgroups (Yang et al., 2010).

In terms of the sociodemographic variables, Frenchspeaking men seemed to be less prone to PSU than those from German-speaking parts. Furthermore, higher achieved educational level was positively associated with PSU, which contrasts with previously published findings (Haug et al., 2015; Kwon, Lee, et al., 2013; Luk et al., 2018). It is possible that those who were better educated were more likely to use their smartphone frequently during their current education or work and thereby developed a constant urge to check their smartphone. Furthermore, their study or work environment potentially allowed them to pursue their urge to check their smartphones constantly (e.g., because they were able to position their smartphone on their desk while working or studying, contrary to someone working at a factory or in some other physical labor).

Despite many strengths of this study (especially the large representative sample), the following limitations should be acknowledged. First, cross-sectional data were used, which disallow causal inferences. Second, C-SURF only included young adult males from Switzerland who were able to converse in either French or German; as such, our results cannot be generalized to the female population, other age groups, or other cultures. Third, all data were based on self-reports and might have been biased. Fourth, more thorough analyses need to be performed in subsequent studies, which should also consider the various types of PSU described in the model from Billieux et al. (2015). Actual usage of smartphones and the motives behind it (e.g., compulsively messaging for reassurance) should be assessed in detail, because such a nuanced view can provide greater insights on the problems associated with and the pathways to PSU than those established through a single PSU construct. The reasons for the unexpected findings (i.e., negative associations between daily cigarette smoking and more than weekly cannabis use with PSU) should also be elaborated in subsequent studies. Fifth, as mentioned above, it must be considered that we used the general term PSU in this study, even though the SAS-SV predominantly assesses an addictive smartphone use. Finally, the pathway model was only used to identify potential predictors for PSU, but not to empirically test the model. Since C-SURF was not specifically tailored to 
test this model, we were limited to use the available predictors. Subsequent studies that aim to empirically test the model of Billieux et al. (2015) should select those predictors that are particularly representative for each pathway and should use suitable statistical methods (e.g., structural equation modeling).

\section{CONCLUSIONS}

The current article provides additional evidence on the different pathways that might lead to PSU. Especially, symptoms of social anxiety (as a risk factor within the excessive reassurance pathway) and ADHD (as risk factor within the impulsive-antisocial pathway) were relevant predictors of PSU in young Swiss men. Furthermore, some evidence of similarities between PSU and behaviors already acknowledged as forms of addiction were detected. For instance, symptoms of ADHD not only predicted PSU (as shown in previous studies) but also substance use disorders (as in the study of Estévez et al., 2016) and other addictive behaviors (e.g., gambling, as in Theule, Hurl, Cheung, Ward, \& Henrikson, 2016). Furthermore, the established relationship between risky RSOD and PSU might be interpreted as evidence of similarities between the two, which supports a theory proposed by Jessor (1991) that risky behaviors tend to cluster.

However, other results of this study might be interpreted as evidence against similarities between PSU and other, already-acknowledged addictive behaviors. First, sensation seeking was not significantly associated with PSU in the multiple regression model, even though this trait has been identified as relevant predictor of substance and gambling addictions (Norbury \& Husain, 2015). Second, the negative associations between frequent cannabis use and cigarette smoking with PSU, as well as the lack of any association between using other illicit drugs besides cannabis and PSU, might be interpreted as evidence of behavioral and biological dissimilarities. This being said, alternative hypotheses must also be considered. Pertaining to sensation seeking, it is possible that a significant association would have been detected if some other PSU-related measure was used, for example, which includes items like phoning while driving or unsafe/risky sexting (Billieux et al., 2015). Concerning the negative association between some substance use variables and PSU, it must be considered that one behavior might act as a substitute for another; for instance, instead of smoking a cigarette, a person might frequently check his/her phone. Finally, the use of illicit drugs (besides cannabis) was not a significant PSU predictor, which might also be because numerous different substances were vastly enveloped within this variable.

The current findings also have practical significance. Knowledge about these newly identified PSU predictors might, for instance, be used to develop tailored interventional programs that address the co-occurrence of certain risky behaviors (e.g., at-risk RSOD and PSU), and particularly focus upon subgroups of individuals who might be particularly prone to PSU (e.g., those with symptoms of ADHD or social anxiety and those who are more educated). Finally, such interventions would also need to ensure that addressing one problem (e.g., decreasing PSU) does not lead to some other compensatory behavior (e.g., frequent cigarette smoking).

Funding sources: C-SURF is supported by the Swiss National Science Foundation (grant number: FN 33CS30_148493).

Authors' contribution: MD conducted the statistical analyses and drafted the manuscript. JS and SH supported the data preparation and analyses. GG is the principal investigator of C-SURF. JS, MPS, GG, DDE, JYC-L, and SH critically reviewed the manuscript. All authors had full access to all data in the study and took responsibility for the integrity of the data and the accuracy of the data analysis.

Conflict of interest: The authors declare no conflict of interest.

\section{REFERENCES}

Aker, S., Şahin, M. K., Sezgin, S., \& Oğuz, G. (2017). Psychosocial factors affecting smartphone addiction in university students. Journal of Addictions Nursing, 28(4), 215-219. doi:10.1097/JAN.0000000000000197

Alhazmi, A. A., Alzahrani, S. H., Baig, M., Salawati, E. M., \& Alkatheri, A. (2018). Prevalence and factors associated with smartphone addiction among medical students at King Abdulaziz University, Jeddah. Pakistan Journal of Medical Sciences, 34(4), 984-988. doi:10.12669/pjms.344.15294

Aluja, A., Rossier, J., García, L. F., Angleitner, A., Kuhlman, M., \& Zuckerman, M. (2006). A cross-cultural shortened form of the ZKPQ (ZKPQ-50-cc) adapted to English, French, German, and Spanish languages. Personality and Individual Differences, 41(4), 619-628. doi:10.1016/j.paid.2006.03.001

Argumosa-Villar, L., Boada-Grau, J., \& Vigil-Colet, A. (2017). Exploratory investigation of theoretical predictors of nomophobia using the Mobile Phone Involvement Questionnaire (MPIQ). Journal of Adolescence, 56, 127-135. doi:10.1016/j. adolescence.2017.02.003

Bech, P., Rasmussen, N. A., Olsen, L. R., Noerholm, V., \& Abildgaard, W. (2001). The sensitivity and specificity of the Major Depression Inventory, using the present state examination as the index of diagnostic validity. Journal of Affective Disorders, 66(2-3), 159-164. doi:10.1016/S0165-0327(00)00309-8

Billieux, J. (2012). Problematic use of the mobile phone: A literature review and a pathways model. Current Psychiatry Reviews, 8(4), 299-307. doi:10.2174/15734001280 3520522

Billieux, J., Maurage, P., Lopez-Fernandez, O., Kuss, D. J., \& Griffiths, M. D. (2015). Can disordered mobile phone use be considered a behavioral addiction? An update on current evidence and a comprehensive model for future research. Current Addiction Reports, 2(2), 156-162. doi:10.1007/s40429015-0054-y

Billieux, J., Van der Linden, M., D’Acremont, M., Ceschi, G., \& Zermatten, A. (2007). Does impulsivity relate to perceived dependence on and actual use of the mobile phone? Applied Cognitive Psychology, 21(4), 527-537. doi:10.1002/acp.1289 
Billieux, J., Van der Linden, M., \& Rochat, L. (2008). The role of impulsivity in actual and problematic use of the mobile phone. Applied Cognitive Psychology, 22(9), 1195-1210. doi:10.1002/ acp. 1429

Boumosleh, J., \& Jaalouk, D. (2017). Depression, anxiety, and smartphone addiction in university students - A cross sectional study. PLoS One, 12(8), e0182239. doi:10.1371/journal.pone. 0182239

Cambridge Dictionary. (2019). Nomophobia. Retrieved from https:// dictionary.cambridge.org/dictionary/english/nomophobia

Chen, B., Liu, F., Ding, S., Ying, X., Wang, L., \& Wen, Y. (2017). Gender differences in factors associated with smartphone addiction: A cross-sectional study among medical college students. BMC Psychiatry, 17(1), 1-9. doi:10.1186/s12888017-1503-Z

Chen, J., Liang, Y., Mai, C., Zhong, X., \& Qu, C. (2016). General deficit in inhibitory control of excessive smartphone users: Evidence from an event-related potential study. Frontiers in Psychology, 7, 1-9. doi:10.3389/fpsyg.2016.00511

Choi, S.-W., Kim, D.-J., Choi, J.-S., Ahn, H., Choi, E.-J., Song, W.-Y., Kim, S., \& Youn, H. (2015). Comparison of risk and protective factors associated with smartphone addiction and Internet addiction. Journal of Behavioral Addictions, 4(4), 308-314. doi:10.1556/2006.4.2015.043

Chung, J. E., Choi, S. A., Kim, K. T., Yee, J., Kim, J. H., Seong, J. W., Seong, J. M., Kim, J. Y., Lee, K. E., \& Gwak, H. S. (2018). Smartphone addiction risk and daytime sleepiness in Korean adolescents. Journal of Paediatrics and Child Health, 54(7), 800-806. doi:10.1111/jpc.13901

Csibi, S., Griffiths, M. D., Cook, B., Demetrovics, Z., \& Szabo, A. (2018). The psychometric properties of the Smartphone Application-Based Addiction Scale (SABAS). International Journal of Mental Health and Addiction, 16(2), 393-403. doi:10.1007/s11469-017-9787-2

Dalrymple, K., Martinez, J., Tepe, E., Young, D., Chelminski, I., Morgan, T., \& Zimmerman, M. (2013). A Clinically Useful Social Anxiety Disorder Outcome Scale. Comprehensive Psychiatry, 54(7), 758-765. doi:10.1016/j.comppsych.2013.02.006

Elhai, J. D., Dvorak, R. D., Levine, J. C., \& Hall, B. J. (2017). Problematic smartphone use: A conceptual overview and systematic review of relations with anxiety and depression psychopathology. Journal of Affective Disorders, 207, 251-259. doi:10.1016/j.jad.2016.08.030

Elhai, J. D., Levine, J. C., \& Hall, B. J. (2019). The relationship between anxiety symptom severity and problematic smartphone use: A review of the literature and conceptual frameworks. Journal of Anxiety Disorders, 62, 45-52. doi:10.1016/ j.janxdis.2018.11.005

Estévez, N., Dey, M., Eich-Höchli, D., Foster, S., Gmel, G., \& Mohler-Kuo, M. (2016). Adult attention-deficit/hyperactivity disorder and its association with substance use and substance use disorders in young men. Epidemiology and Psychiatric Sciences, 25(3), 255-266. doi:10.1017/S2045796015000360

Gmel, G., Akre, C., Astudillo, M., Bähler, C., Baggio, S., Bertholet, N., Clair, C., Cornuz, J., Daeppen, J.-B., Deline, S., Dermota, P., Dey, M., Dupuis, M., Estévez, N., Foster, S., Gaume, J., Haug, S., Henchoz, Y., Kuendig, H., Mohler-Kuo, M., N'Goran, A., Schaub, M., Studer, J., Suris, J.-C., \& Wang, J. (2015). The Swiss cohort study on substance use risk factors - Findings of two waves. Sucht, 61(4), 251-262. doi:10.1024/0939-5911.a000380
Haug, S., Castro, R. P., Kwon, M., Filler, A., Kowatsch, T., \& Schaub, M. P. (2015). Smartphone use and smartphone addiction among young people in Switzerland. Journal of Behavioral Addictions, 4(4), 299-307. doi:10.1556/2006.4. 2015.037

Hoyle, R. H., Stephenson, M. T., Palmgreen, P., Lorch, E. P., \& Donohew, R. L. (2002). Reliability and validity of a brief measure of sensation seeking. Personality and Individual Differences, 32(3), 401-414. doi:10.1016/S0191-8869(01) 00032-0

IBM Corp. (2013). IBM SPSS for Windows, version 22.0. Armonk, NY: IBM Corp.

Jessor, R. (1991). Risk behavior in adolescence: A psychosocial framework for understanding and action. The Journal of Adolescent Health, 12(8), 597-605. doi:10.1016/1054139X(91)90007-K

Jun, S. (2016). The reciprocal longitudinal relationships between mobile phone addiction and depressive symptoms among Korean adolescents. Computers in Human Behavior, 58, 179-186. doi:10.1016/j.chb.2015.12.061

Kessler, R. C., Adler, L. A., Ames, M., Demler, O., Faraone, S., Hiripi, E., Howes, M. J., Jin, R., Secnik, K., Spencer, T., Ustun, T. B., \& Walters, E. E. (2005). The World Health Organization Adult ADHD Self-Report Scale (ASRS): A short screening scale for use in the general population. Psychological Medicine, 35(2), 245-256. doi:10.1017/ S0033291704002892

Kessler, R. C., Adler, L. A., Gruber, M. J., Sarawate, C. A., Spencer, T., \& Van Brunt, D. L. (2007). Self-reported utilization of mental health services in the adult German population. International Journal of Methods in Psychiatric Research, 16(2), 52-65. doi:10.1002/mpr.208

Kim, Y., Jeong, J. E., Cho, H., Jung, D. J., Kwak, M., Rho, M. J., Yu, H., Kim, D. J., \& Choi, I. Y. (2016). Personality factors predicting smartphone addiction predisposition: Behavioral inhibition and activation systems, impulsivity, and self-control. PLoS One, 11(8), 1-15. doi:10.1371/ journal.pone.0159788

Kwon, M., Kim, D.-J., Cho, H., \& Yang, S. (2013). The Smartphone Addiction Scale: Development and validation of a short version for adolescents. PLoS One, 8(12), e83558. doi:10.1371/journal.pone.0083558

Kwon, M., Lee, J. Y., Won, W. Y., Park, J. W., Min, J. A., Hahn, C., Gu, X., Choi, J.-H., \& Kim, D. J. (2013). Development and validation of a Smartphone Addiction Scale (SAS). PLoS One, 8(2), e56936. doi:10.1371/journal.pone.0056936

Lin, Y. H., Chiang, C. L., Lin, P. H., Chang, L. R., Ko, C. H., Lee, Y. H., \& Lin, S. H. (2016). Proposed diagnostic criteria for smartphone addiction. PLoS One, 11(11), 1-11. doi:10.1371/ journal.pone. 0163010

Luk, T. T., Wang, M. P., Shen, C., Wan, A., Chau, P. H., Oliffe, J., Viswanath, K., Chan, S. S., \& Lam, T. H. (2018). Short version of the Smartphone Addiction Scale in Chinese adults: Psychometric properties, sociodemographic, and health behavioral correlates. Journal of Behavioral Addictions, 7(4), 1157 1165. doi:10.1556/2006.7.2018.105

Myers, R. (1990). Classical and modern regression with applications (2nd ed.). Boston, MA: Duxbury.

Norbury, A., \& Husain, M. (2015). Sensation-seeking: Dopaminergic modulation and risk for psychopathology. Behavioural Brain Research, 288, 79-93. doi:10.1016/j.bbr.2015.04.015 
Olsen, L. R., Jensen, D. V., Noerholm, V., Martiny, K., \& Bech, P. (2003). The internal and external validity of the Major Depression Inventory in measuring severity of depressive states. Psychological Medicine, 33(2), 351-356. doi:10.1017/ S0033291702006724

Oxford Dictionary. (2018). Smartphone. Retrieved October 25, 2018, from https://en.oxforddictionaries.com/definition/smartphone

Panova, T., \& Carbonell, X. (2018). Is smartphone addiction really an addiction? Journal of Behavioral Addictions, 7(2), 252-259. doi:10.1556/2006.7.2018.49

Sánchez-Martínez, M., \& Otero, A. (2009). Factors associated with cell phone use in adolescents in the community of Madrid (Spain). CyberPsychology \& Behavior, 12(2), 131-137. doi:10.1089/cpb.2008.0164

Theule, J., Hurl, K. E., Cheung, K., Ward, M., \& Henrikson, B. (2016). Exploring the relationships between problem gambling and ADHD. Journal of Attention Disorders. Advance online publication. doi:10.1177/1087054715626512
Thomée, S., Härenstam, A., \& Hagberg, M. (2011). Mobile phone use and stress, sleep disturbances, and symptoms of depression among young adults - A prospective cohort study. BMC Public Health, 11(1), 66. doi:10.1186/1471-2458-11-66

Vahedi, Z., \& Saiphoo, A. (2018). The association between smartphone use, stress, and anxiety: A meta-analytic review. Stress and Health, 34(3), 347-358. doi:10.1002/smi.2805

Yang, Y. S., Yen, J. Y., Ko, C. H., Cheng, C. P., \& Yen, C. F. (2010). The association between problematic cellular phone use and risky behaviors and low self-esteem among Taiwanese adolescents. BMC Public Health, 10(1), 217. doi:10.1186/ 1471-2458-10-217

Zheng, F., Gao, P., He, M., Li, M., Wang, C., Zeng, Q., Zhou, Z., Yu, Z., \& Zhang, L. (2014). Association between mobile phone use and inattention in 7102 Chinese adolescents: A populationbased cross-sectional study. BMC Public Health, 14(1), 1022. doi:10.1186/1471-2458-14-1022 